\title{
Adaptive Routing in Mobile Opportunistic Networks
}

\author{
Jani Lakkakorpi \\ Aalto University, Comnet \\ Espoo, Finland \\ jani.lakkakorpi@iki.fi
}

\author{
Mikko Pitkänen \\ Helsinki Institute of Physics \\ Helsinki, Finland \\ mikko.pitkanen@hip.fi
}

\author{
Jörg Ott \\ Aalto University, Comnet \\ Espoo, Finland \\ joerg.ott@tkk.fi
}

\begin{abstract}
In this paper, we study how to adapt the routing according to dynamic network conditions in wireless ad hoc networks. We present a method that dynamically chooses routing agent between ad hoc on-demand distance vector routing protocol (AODV) with TCP (endto-end transport) and delay-tolerant networking (DTN) routing and bundle protocol (hop-by-hop transport). We use simulations to confirm that DTN routing and the bundle protocol leads to significantly shorter end-to-end delays and higher message delivery ratios than AODV and end-to-end TCP when the wireless node density is low. However, with high node density, DTN routing, especially epidemic routing, suffers from multiple bundle copies and simultaneous transmissions that lead into collisions and retransmissions at the wireless link (MAC) layer. Thus, we propose a simple adaptive scheme that uses only local information to transmit the messages from source to destination using either AODV or DTN routing, depending on current node density, message size, and path length to destination.
\end{abstract}

\section{Categories and Subject Descriptors}

C.2.2 [Computer-Communication Networks]: Network Protocols

\section{General Terms}

Algorithms, Design, Performance

\section{Keywords}

DTN, AODV, ns-2

\section{INTRODUCTION}

Mobile ad hoc networks (MANETs) [10] are wireless networks that can function without any supporting infrastructure, e.g., base stations. Several use cases have been suggested for MANETs, including wireless sensor networks and military communication systems. The increasing wireless capabilities of devices carried by people have increased interest to use ad hoc networking also between the mobile users. However, highly dynamic nature of the

Permission to make digital or hard copies of all or part of this work for personal or classroom use is granted without fee provided that copies are not made or distributed for profit or commercial advantage and that copies bear this notice and the full citation on the first page. To copy otherwise, to republish, to post on servers or to redistribute to lists, requires prior specific permission and/or a fee.

MSWiM'10, October 17-21, 2010, Bodrum, Turkey.

Copyright 2010 ACM 978-1-4503-0274-6/10/10 ...\$10.00. network formed by the users imposes several challenges for the communication.

In MANETs, the wireless nodes relay messages to each other using a common routing protocol. The routing protocol requires that there exists an end-to-end path from the message source node to the destination node. If the path does not exist, message transmission is postponed until a valid path becomes available. Based on earlier work [20,9], we assume that this condition is difficult to meet if the network of nodes is very sparse or the nodes are highly mobile. These conditions often occur in network that is set up between mobile users, thus, this paper investigates how to adapt routing to situations where these conditions occur.

Delay-tolerant networking (DTN) [2] architecture offers support for communication scenarios where nodes are sparse and the contacts between them are short-lived, e.g., due to high node mobility. The DTN approach allows the intermediate nodes to store messages for extended period of time (i.e., carry), and to deliver messages towards destination when opportunity to forward a message becomes available. Thus, in contrary to MANET approach, the DTNs can deliver messages also when instantaneous end-to-end path between the nodes does not exist. However, many DTN routing protocols aim to ensure delivery by creating multiple message copies, which can lead to congestion and decreased performance, especially in dense networks.

In this paper, we show how to adaptively choose between DTN and MANET routing to adjust to network conditions that are dynamic in terms of node density and velocity. To evaluate the proposed approach we use a simulation model that closely follows real world use cases. Our modeling is novel in two aspects. On the one hand, we model the effect of wireless physical layer congestion, which is often omitted in DTN simulations. On the other hand, the simulations are conducted with synthetic and real life mobility traces that model the proposed usage scenario more closely than than many earlier MANET studies. Our simulations cover several network scenarios and confirm that it is beneficial to integrate MANET and DTN routing so that the method for the message delivery is chosen for each message adaptively on a case-by-case basis when sending the message.

The rest of this paper is organized as follows. Section 2 summarizes the ad hoc on-demand distance vector routing protocol (AODV), gives an overview on DTNs and the bundle protocol and covers earlier related work. Section 3 shows simulation results to confirm our initial assumptions and motivates the adaptive routing approach. Section 4 introduces the proposed adaptive routing mechanism for mobile DTNs, and shows additional simulation results on the performance of the adaptive approach. Finally, Section 5 concludes the paper with a discussion. 


\section{BACKGROUND AND RELATED WORK}

Early research, covered in the first sub-section, aimed to establishing paths between the end points of the communication. The second subsection explains DTN routing, which overcomes inability to establish paths by using contacts between the nodes for hopby-hop delivery. The third sub-section covers other relate work on the field.

\subsection{AODV Routing}

AODV protocol is designed especially for routing in MANETs. It is an on-demand algorithm, i.e., a path between the nodes is set up only when it is requested by the node willing to originate a message. Each path is maintained only as long as its originator needs it or the path breaks, e.g., due to an intermediate node moving away. In the following paragraph, we summarize the basics of AODV unicast routing according to work by Perkins et $a l .[12,11]$.

Whenever a route is needed between two nodes, the originator node broadcasts a route request (RREQ) message across the network (Figure 1a). Nodes that receive the RREQ then update their information related to the originator node in question and set backward pointers to the originator node in their route tables. Reverse routes (Figure 1b) are set up in order to forward the route reply (RREP) messages back to the originator from the destination or from an intermediate node having a route to the destination [11]. A node that receives the RREQ message may unicast an RREP message back to the originator node if it is the destination or if it has a fresh enough route to the destination. Otherwise, the node will rebroadcast the RREQ. All nodes keep track of those RREQs they have already seen, and in case of previously received RREQs the duplicates are silently discarded.

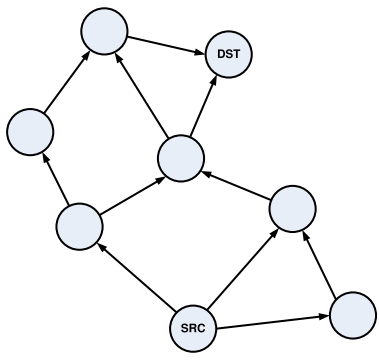

(a) RREQ propagation.

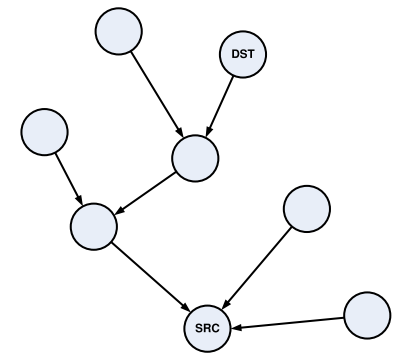

(b) Reverse route entries.
Figure 1: AODV route discovery.

As the RREP propagates back to the originator node, all nodes along the path set up forward pointers to the destination node. Then, the originator node will be able to send data packets to the destination node after receiving the RREP. If the originator node later happens to receive a RREP containing a greater sequence number or a RREP with the same sequence number but a smaller hop count, it may update its routing information for that destination and start using the better route.

Routes are maintained as long as they remain active, i.e., as long as there is frequent enough data traffic to the destination. When traffic to a destination stops, the route will time out and eventually it will be deleted from the route table. If a link break occurs while the route is still active, a route error (RERR) message to the originator node is sent by the node that is in the end closer to the originator node. If the originator node still needs routes to these destinations, it reinitiates route discovery and tries to establish a path.

\subsection{DTN and the Bundle Protocol}

DTN [2] architecture introduces a bundle protocol [15], which offers transport services for applications. The bundles are usually significantly larger than IP packets allowing to create semantically self-contained messages that enable complete application interactions within a single round trip time.

Instead of relying on end-to-end communication, the bundles are transmitted based on hop-by-hop reliability. Any suitable protocol (such as TCP or UDP) can be used for transferring a bundle over a single hop. Bundles have a certain lifetime, also known as time-to-live (TTL), after which they expire and are deleted from intermediate nodes. Bundle retransmission can take place either at the originating node or at an intermediate node, which has obtained the custody of the bundle in a process called custody transfer.

Several different routing protocols can be applied for the bundles. Some of the protocols create multiple copies of message to increase reliability of reaching to the destination. Epidemic routing [18] works as follows: when node A receives a Hello message, containing summary about the buffer contents, from its neighbor node $\mathrm{B}$, node $\mathrm{A}$ immediately compares the Hello message summary against its own buffer and will send to node B all the bundles whose identifiers were not included in the Hello message.

Spray and wait routing [16], limits the number of bundle copies to a fixed value. Binary spray and wait works as follows: any node A that has more than a single bundle token, and encounters another node $\mathrm{B}$ (with no tokens), hands over to node $\mathrm{B}$ half of the tokens. When node A is left with only a single token, the corresponding bundle can be forwarded to destination only, not to relay nodes anymore.

Figure 2 illustrates how a bundle finds its way from source to destination in a wireless ad hoc network using DTN routing, e.g., spray and wait, and the bundle protocol. At $t=T$, node B is close enough to the destination node and the bundle can be transmitted to its final destination.

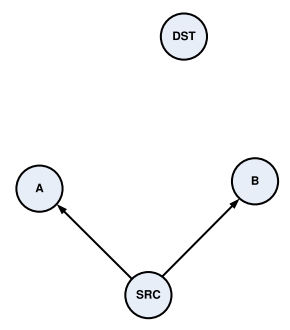

(a) $t=0$.

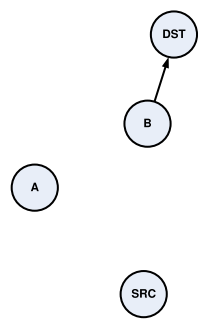

(b) $t=T>0$.
Figure 2: Bundle dissemination in a wireless ad hoc network.

Independent of the routing protocol, when the bundle is received in the destination, the node may generate a return receipt to the source node. In our case, the return receipts also act as antipackets. We use the VACCINE antipacket mechanism [3], where bundles are deleted and nodes get immunity, i.e. they stop accepting the bundle as the return receipt is forwarded towards the bundle source. Whatever is used for the bundle routing scheme, antipackets are always spread using epidemic routing [19]. Antipackets will eventually expire, just like the bundles.

\subsection{Related Work}

The performance of end-to-end versus hop-by-hop transport under intermittent connectivity has been studied by Heimlicher et 
al. [5]. According to the analytical model derived by the authors, end-to-end may surprisingly perform better (higher packet delivery ratio) under long disruption periods than hop-by-hop. This is reportedly due to larger retransmission interval in the end-to-end transport scheme. However, simulations where the performance of TCP NewReno was compared to that of Store-And-Forward Transport (SAFT) [4] did not agree with the analytical results. In our opinion, this may be due to limitations of the analytical model, e.g., node mobility is not captured in the model.

There exists earlier proposals [20,9] for integrating DTN and MANET routing. A hybrid DTN-MANET routing protocol, HYMAD, was introduced by Whitbeck et al. [20]. HYMAD uses DTN between disjoint groups of nodes while MANET routing is used within the groups. Simulation results show that HYMAD is able to outperform multi-copy spray and wait DTN routing protocol, both in terms of delivery ratio and delay. HYMAD, however, works based on assumption that the DTN nodes form groups and seems more suitable for networks where such grouping occurs.

Another hybrid scheme that combines AODV and DTN routing is presented by Ott et al. [9]. The presented approach maintains end-to-end semantics whenever possible,. When the end-to-end connections break, the DTN-based communication is used as an option. The approach is similar to one discusses in this paper that the communication application can choose the used protocol. However, this paper presents significantly more extensive evaluation in realistic mobile networks.

Delay Tolerant IP Networking [7] proposes IP based networking for long delay environments. While the proposed solution seems suitable for delivering moderately small data units, big files would produce a large number of fragments, which renders the approach infeasible in our scenarios.

Sadagopan et al. [14] presented a study of path duration in MANET. Their findings show that under various mobility models duration for paths longer than two hops depends on relative speed of the node, transmission range of the node, and number of hops to destination. Similar to our approach, the finding shows that the path characteristics can be estimated based on locally available information only.

\section{PERFORMANCE EVALUATION}

We start our performance evaluation by experimenting with both DTN and AODV routing with wide range of simulation and routing parameters. These results then serve as an input for our adaptive algorithm, which is presented in section 4. Our simulations use several extensions to $n s 2$ simulator that allow to study DTN routing together with accurate models for radio links. Our initial simulations build on top of synthetically generated random waypoint (RWP) models

\subsection{Mobility Models}

We chose random waypoint to be our main mobility model since this model is well understood and it is easy to generate scenarios with different network densities and node velocities. We generate random waypoint node mobility using the setdest program (part of ns-allinone package [17]). We have 40 or 80 mobile nodes that select a random direction and a random (uniformly distributed) speed at random times. Maximum speed is either $2.5 \mathrm{~m} / \mathrm{s}$ or $20 \mathrm{~m} / \mathrm{s}$, depending on simulated case, and pause length is two seconds. We always pause before choosing a new direction and a new speed. Area size ranges from $10 \mathrm{~m}$ times $10 \mathrm{~m}$ to $2000 \mathrm{~m}$ times $2000 \mathrm{~m}$. In our random waypoint scenarios, the simulation time is always 5000 seconds.

Two more realistic mobility models are explained in section 4 after introducing our adaptive routing mechanism, which uses the result obtained in this section as an input.

\subsection{Traffic Model}

We use a simple traffic model in which each node sends a $10 \mathrm{kB}$, $100 \mathrm{kB}$ or $500 \mathrm{kB}$ message at a random time with 200 second intervals $[t, t+200 s]$ to another, randomly selected, node. In the AODV case, we use TCP (commonly used NewReno variant) with default settings to transfer the messages through an end-to-end connection. In the DTN case, the bundles are fragmented to 1500-byte IP packets before sending them to the MAC layer. A retransmission mechanism providing reliable delivery of IP packets is implemented, too.

\subsection{DTN Model}

DTN nodes advertise their buffer content to each other every 100 ms by sending Hello messages. In our simulations, this message has enough room for the identifiers of buffered bundles and return receipts. A bundle can be generated only if the node has sufficient buffer space. We have chosen to use $100 \mathrm{MB}$ buffer space, which does not become a bottleneck in the simulations. Bundle lifetime is set to 750 seconds, after which all copies of the bundle will be deleted. If the sending node does not receive a return receipt within 1000 seconds, it will retransmit the bundle ${ }^{1}$. Antipacket lifetime is the minimum of retransmission timeout (1000 seconds) less bundle forwarding time and bundle lifetime (750 seconds). Antipackets and Hello messages are small in size. We run all DTN simulations both with epidemic routing and binary spray and wait with 16 message copies.

Even though several routing protocols exists for DTNs, it is desirable that a uniform routing scheme is used within the network to avoid harmful routing interaction. The approach proposed in this paper makes decision between using AODV or DTN for message delivery. In case the DTN approach is chosen, a uniform DTN routing scheme is always used for message delivery.

\subsection{Wireless Channel Model}

We use a realistic wireless channel model from dei80211mr library [8], which is now a part of the ns-allinone package, and provides more realistic modelling than the default model in $n s 2$. The library provides support for different transmission rates, modulation methods and coding schemes that are defined in the IEEE $802.11 \mathrm{~b} / \mathrm{g}$ standards.

A signal-to-interference-and-noise ratio (SINR) based packet level error model is introduced. The reception threshold (RXThresh_) variable, which is used in the default 802.11 implementation, has been removed. Instead, packet error rate (PER) is used to determine random packet losses. PER is calculated using pre-determined curves of PER vs. SINR and packet size. SINR is calculated using received signal strength, noise, and interference. Interference is calculated using a gaussian model to account for all transmissions that happen simultaneously to the one which is considered for reception. Finally, strength of noise is fixed in all simulations.

The capture model, i.e., the determination of whether a packet can be received when there are other concurrent transmissions, is embedded in the aforementioned interference model.

The 802.11 parameters are chosen to model $802.11 \mathrm{~g}$ and they are listed in Table I. Noise is set according to

$$
P n=k T B,
$$

where $k$ is Boltzmann's constant $(1.38 \mathrm{e}-23 \mathrm{~J} / \mathrm{K}), T$ is room temperature $(290 \mathrm{~K})$, and $B$ is bandwidth $(2.437 \mathrm{GHz})$. With the selected parameters, node transmission range will be 66-130 meters,

${ }^{1}$ In the AODV/TCP case, TCP takes care of the retransmissions. 
Table 1: IEEE 802.11g related simulation parameters.

\begin{tabular}{|c||c|}
\hline Parameter & Value \\
\hline \hline noise_ & $9.75 \mathrm{e}-12 \mathrm{~W}$ \\
\hline CSThresh__ & $1 \mathrm{e}-10 \mathrm{~W}$ \\
\hline Pt_ & $0.0178 \mathrm{~W}$ \\
\hline freq_ & $2.437 \mathrm{e} 6 \mathrm{~Hz}$ \\
\hline L__ $_{-}$ & 1.0 \\
\hline useShortPreamble_ & true \\
\hline gSyncInterval_ & $0.00001 \mathrm{~s}$ \\
\hline CWMin__ & 15 \\
\hline CWMax_ & 1023 \\
\hline RTSThreshold_ & $0 \mathrm{~B}$ \\
\hline ShortRetryLimit_ & 7 \\
\hline LongRetryLimit_ & 4 \\
\hline SlotTime_ & $0.000009 \mathrm{~s}$ \\
\hline SIFS_ & $0.000016 \mathrm{~s}$ \\
\hline
\end{tabular}

depending on the current modulation and coding scheme (MCS) of the connection. The better the MCS, the higher the transmission rate. However, the better the MCS, the smaller the transmission range.

\subsection{RWP Mobility: 40 Nodes, Small Messages}

The evaluation starts with random waypoint simulations with 40 mobile nodes and $10 \mathrm{kB}$ message size. Figure 3 shows that epidemic routing gives the best performance in terms of delivery ratio (Figure 3a) and delay (Figure 3b) when the area size is bigger than $500 \mathrm{~m}$ times $500 \mathrm{~m}$. Neither buffer space (Figure 3d) nor bandwidth is seen as a bottleneck. The bandwidth bottleneck can be observed from head-of-line blocking period durations (Figure 3e): as long as the interface queue is non-empty, forwarding of new bundles cannot start. Also binary spray and wait performs better than AODV when the network is sparse. With more dense cases, AODV gives the best results according to initial assumptions. Further observation is that when the area size grows, the delays grow, too, and the delivery ratios decrease. When the network is really sparse, AODV delivery ratio falls below 60 percent as seen in Figure 3a. This happens because the paths from source to destination are active only for short periods of time and break during message transfers.

The results without antipacket (No AP in figure) mechanism (such as VACCINE) illustrate that the lack of bandwidth is a bottleneck in dense DTN scenarios (Figure 3e). This can be seen as high delay. Buffer space can also become a bottleneck as the bundles are stored in intermediate nodes until their TTLs expire.

Figure 4 shows results with slow-velocity nodes, maximum speed is $2.5 \mathrm{~m} / \mathrm{s}$ instead of $20 \mathrm{~m} / \mathrm{s}$. We observe that both AODV and DTN results get worse when the network gets more sparse. DTN seems to suffer from slow velocity more than AODV. This can be explained by that, when the nodes are moving slow, it is harder

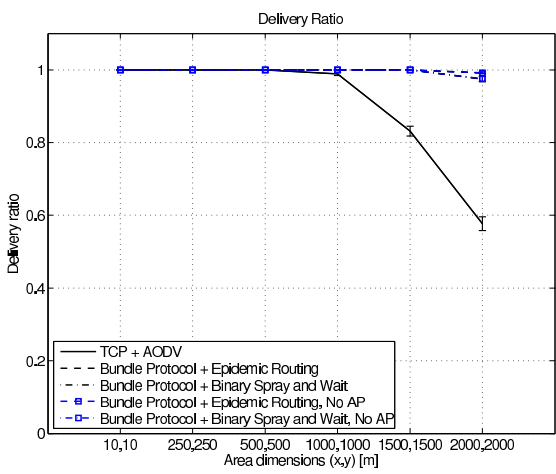

(a) Delivery ratio.

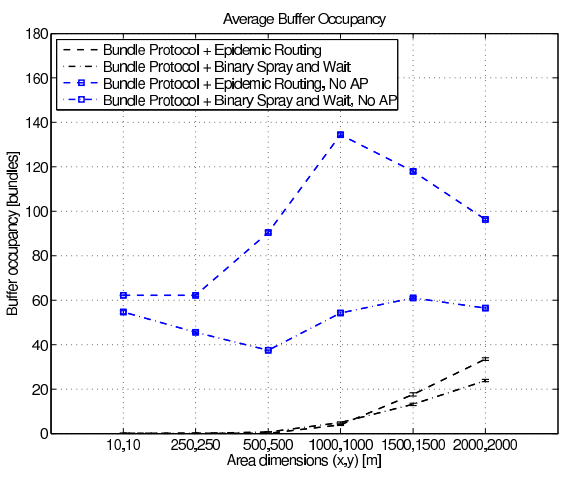

(d) Average buffer occupancy.

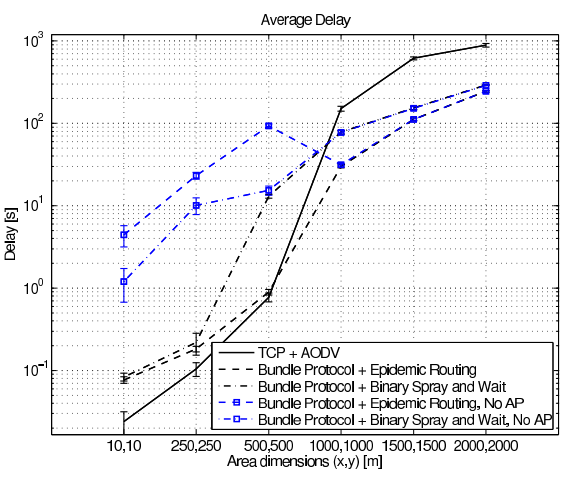

(b) Average delay.

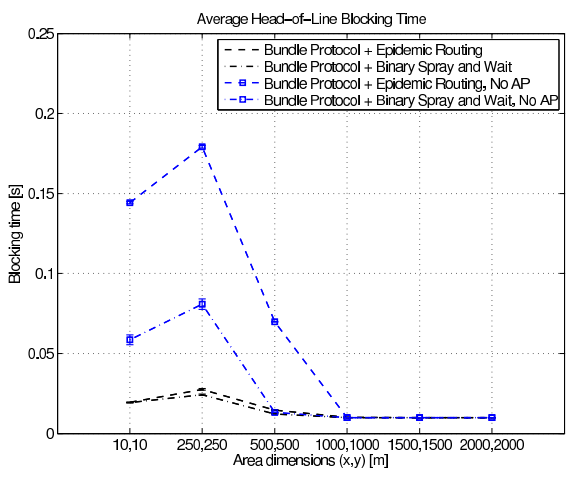

(e) Average head-of-line blocking.

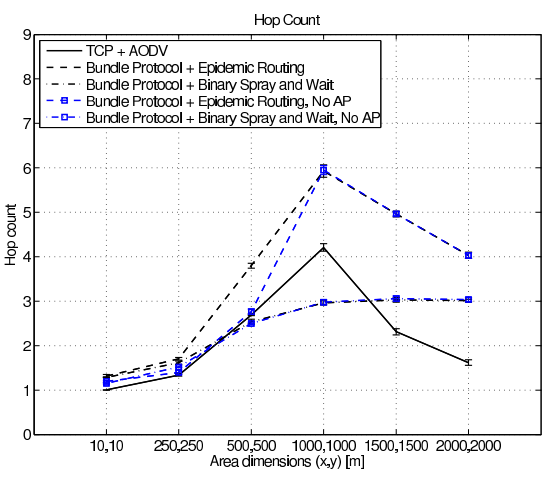

(c) Average hop count.

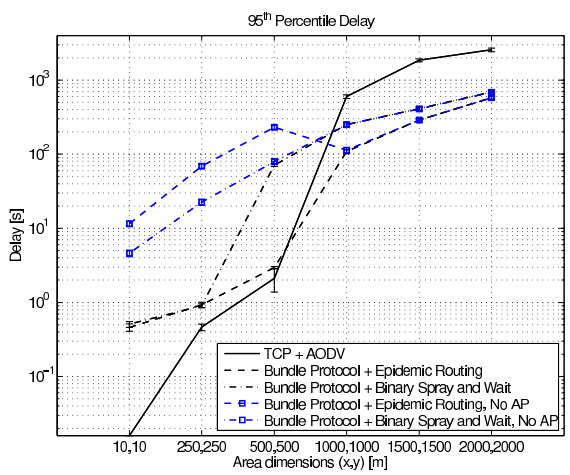

(f) 95th percentile delay.

Figure 3: Random waypoint: 40 nodes, $10 \mathrm{kB}$ messages, fast mobility (max. $20 \mathrm{~m} / \mathrm{s}$ ). 
to find new neighbors. However, DTN still clearly outperforms AODV with the low node densities.

\subsection{RWP Mobility: 80 Nodes, Small Messages}

Figure 5 shows results with larger node population (80 mobile nodes) and $10 \mathrm{kB}$ message size. We can see AODV clearly outperforming DTN when the network is dense. This seems intuitive, as it becomes much easier to setup and maintain paths from source to destination. Epidemic routing produces so much traffic that the network becomes congested: this leads into collisions and retransmissions at the wireless link layer. However, this is not a problem in the $10 \mathrm{~m}$ times $10 \mathrm{~m}$ scenario as then all nodes are within each other's range and thus only one copy of a bundle needs to be sent. On the contrary, when the area size grows, congestion disappears as the nodes have fewer neighbors. Also the binary spray and wait (with 16 bundle copies) routing performs well in this scenario.

\subsection{RWP Mobility: 40 Nodes, Large Messages}

Figures 6 and 7 show the results obtained with 40 mobile nodes and large messages (100 or $500 \mathrm{kB}$ in size). We observe that the performance of epidemic routing collapses when the area size is between $500 \mathrm{~m}$ times $500 \mathrm{~m}$ and $1000 \mathrm{~m}$ times $1000 \mathrm{~m}$. The spray and wait, however, maintains good delivery ratio with $100 \mathrm{kB}$ bundles, which suggests that the epidemic routing causes excess congestion with larger bundles. In the case of $500 \mathrm{kB}$ bundles both DTN routing protocols suffer from low delivery performance, which follows from bundle size exceeding the transfer capacity of available opportunistic contacts. Large message size does not have similar impact on AODV but the performance degradation in relation to network density is similar to that with the small $10 \mathrm{kB}$ message size.

\section{ADAPTIVE ROUTING IN MOBILE DTNS}

Our initial observations clearly confirm how using varying routing mechanisms is more suitable in different communication scenarios. We could not find a single routing approach, which would have been the best in all scenarios. This motivates us to explore how to combine DTN and AODV routing to achieve increased message delivery success together with resource conservation. We especially want to overcome the well-known fact that AODV and other MANET routing protocols use less network resources (buffer space and bandwidth) than DTN routing protocols. Especially epidemic routing is problematic as it uses flooding for forwarding the messages.

Our approach is to choose in the sending node whether to use DTN (e.g., epidemic or spray and wait) or AODV for message delivery. The benefit of the approach is that both routing protocols can remain untouched, and intermediate node need to support only pure DTN or AODV functionality. The decision on which protocol to use for transmitting a given message from source to destination is made on application level.

Figure 8 shows the main parameters we use for choosing the used

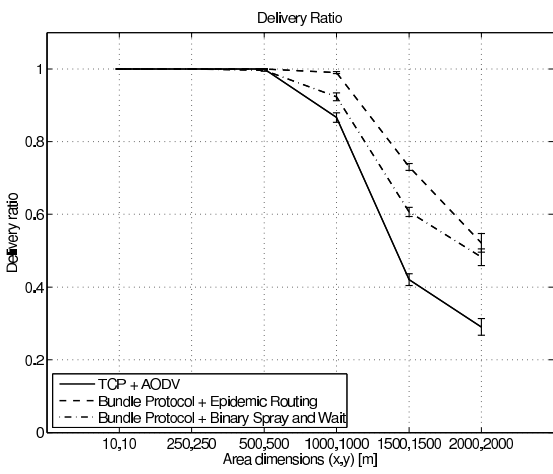

(a) Delivery ratio.

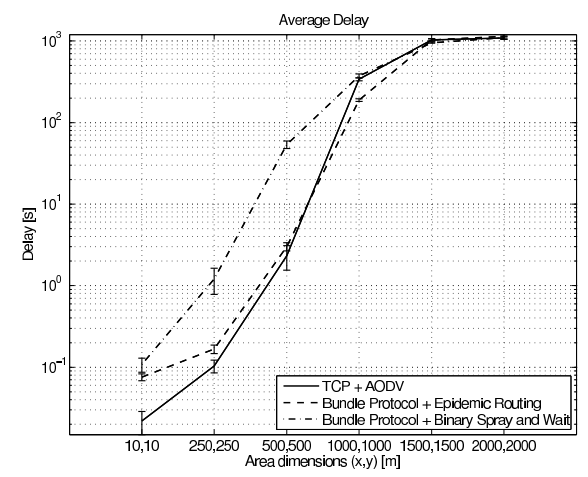

(b) Average delay.

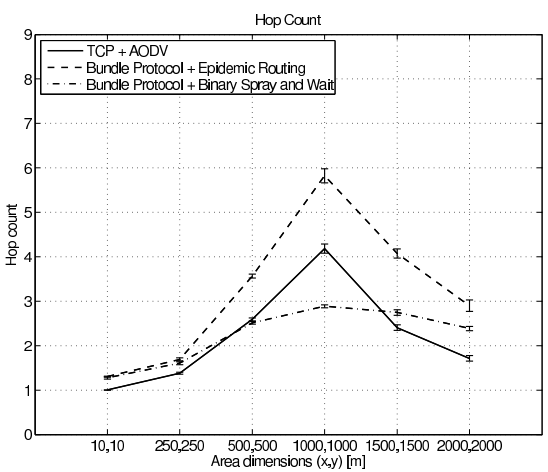

(c) Average hop count.

Figure 4: Random waypoint: 40 nodes, $10 \mathrm{kB}$ messages, slow mobility (max. $2.5 \mathrm{~m} / \mathrm{s}$ ).

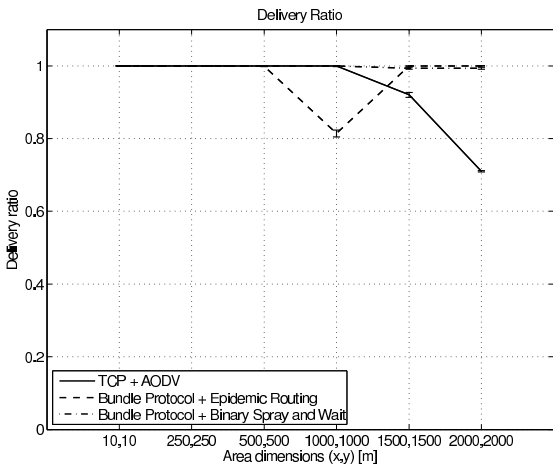

(a) Delivery ratio.

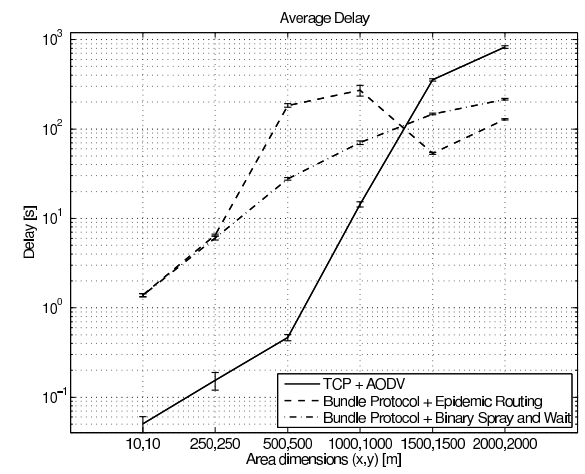

(b) Average delay.

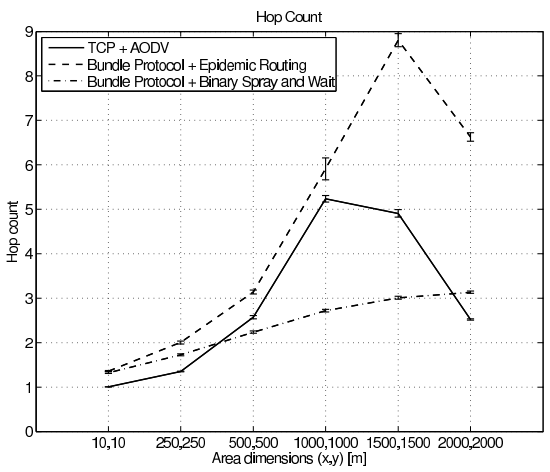

(c) Average hop count.

Figure 5: Random waypoint: 80 nodes, $10 \mathrm{kB}$ messages, fast mobility (max. $20 \mathrm{~m} / \mathrm{s}$ ). 


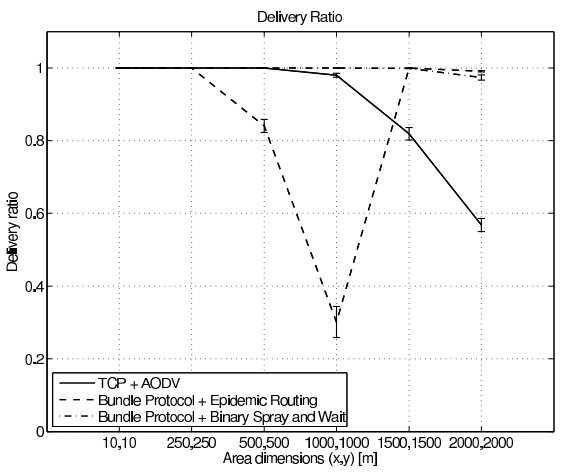

(a) Delivery ratio.

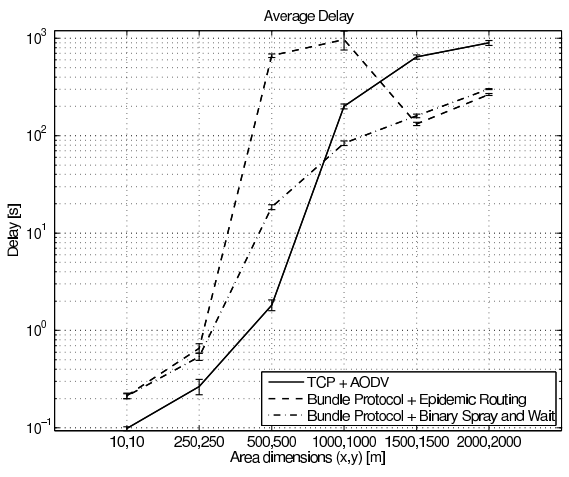

(b) Average delay.

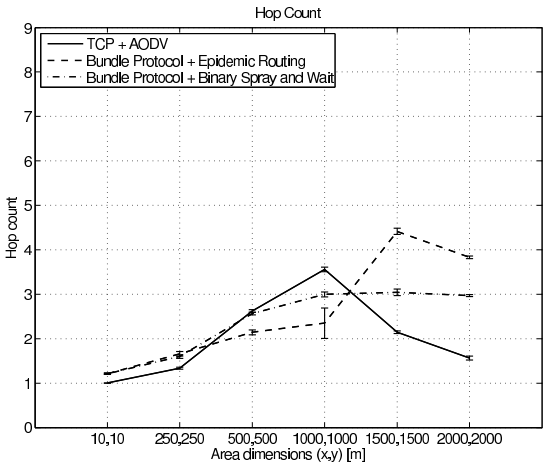

(c) Average hop count.

Figure 6: Random waypoint: 40 nodes, $100 \mathrm{kB}$ messages, fast mobility (max. $20 \mathrm{~m} / \mathrm{s}$ ).

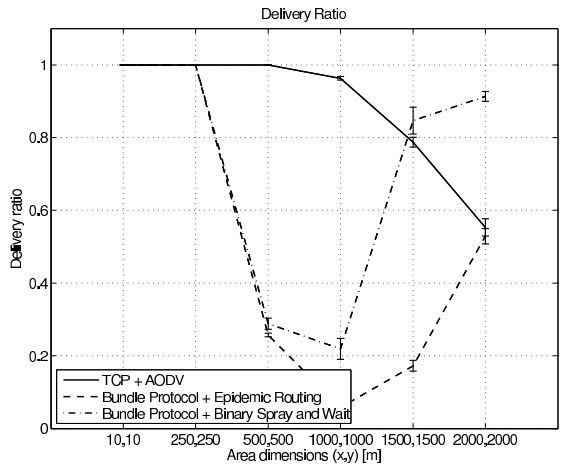

(a) Delivery ratio.

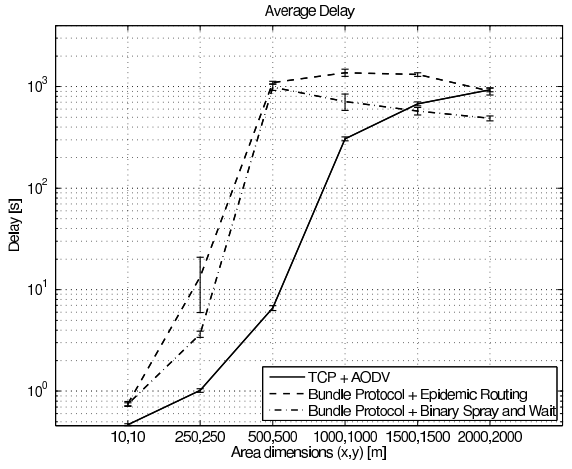

(b) Average delay.

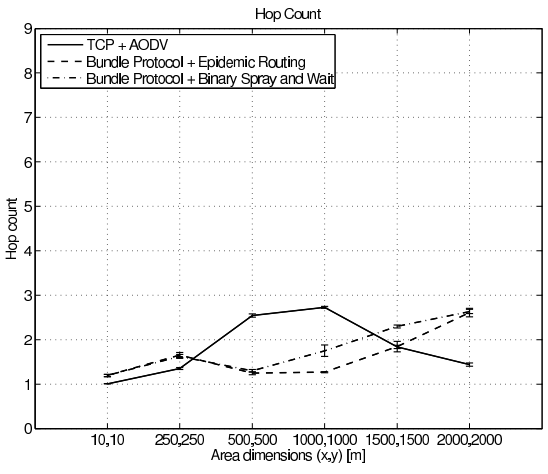

(c) Average hop count.

Figure 7: Random waypoint: 40 nodes, $500 \mathrm{kB}$ messages, fast mobility (max. $20 \mathrm{~m} / \mathrm{s}$ ).

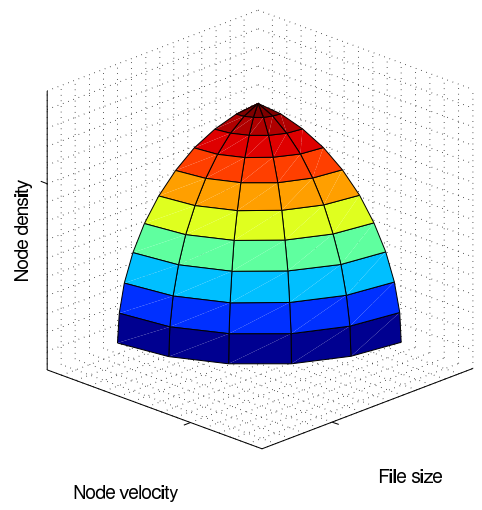

Figure 8: DTN (inside) and MANET (outside) areas.

routing approach. These can be seen in three main axes; network density, node velocity and message size. The first parameter can be inferred implicitly from mobility and contact patterns, and the other two, message size and node velocity can be obtained from local information in the sending node. Assumption that it takes more time to transmit bigger messages requires the paths to remain stable longer than with smaller messages, thus large messages are not favorable for MANET. At first, this may seem to be in contrast to what we saw in Figures 6 and 7, however, the drop in DTN routing performance is more due to high network density than large message size.

Moreover, the higher the node velocity, the shorter the route lifetime. Thus, it is seems suitable to switch to DTN routing when the message is big and/or the node is moving fast. Naturally, we need to find this switching point. In order to obtain the network density, we make the assumption that all nodes in the network have the same communication range. Then, based on the number of nodes that we are currently communicating with, we can calculate the node density. Based on several earlier studies, we believe that when the network is dense enough, it makes sense to use MANET routing as it leads to lower delays and the load on the network is substantially lower than with DTN routing.

Based on our observations, we craft Algorithm 1, which follows the logic behind our routing decisions: when the network is sparse, nodes are moving fast, or messages are big, we use DTN routing. Based on the results presented in the following section, we have derived a switching point: when the estimated message sending time is less than the estimated path lifetime, we use MANET routing and DTN routing otherwise. The following subsections evaluate the working of this algorithm.

We have parametrized the algorithm by setting $b w$ to $5 \mathrm{Mbps}$ [13] and $d_{\text {ref }}$ to 8.5. The latter constant based is based on our random waypoint simulations, described in the previous section. 


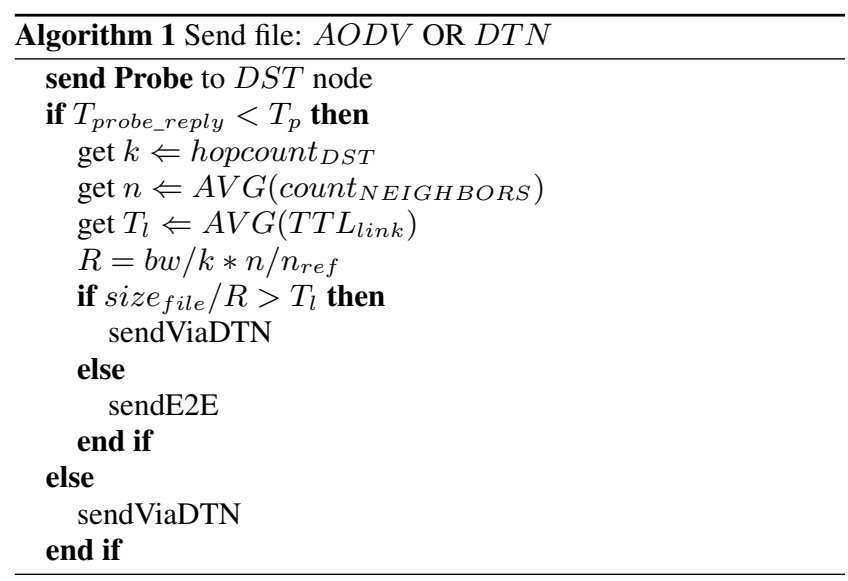

Morever, exponentially averaged link lifetime $\left(T_{l}\right)$ is used as an estimate for the path lifetime. Hop count $(k)$ is obtained from the probe packet ${ }^{2}$ reply while the exponentially ${ }^{3}$ averaged number of neighbors $(d)$ and the averaged link lifetime are obtained from the DTN agent. As we use only information that we have gathered ourself, the algorithm is trustworthy.

\subsection{RWP Mobility}

Based on our observations in section 3, we choose to use AODV when the network density is above $40 /(500 \mathrm{~m} * 500 \mathrm{~m})$ and epidemic routing otherwise. In order to obtain the network density, we assume that the communication range of all nodes is $130 \mathrm{~m}$ and then, based on the number of nodes that we are communicating with, we can calculate the node density: $n /\left(\pi * r^{2}\right)$, where $n$ is the number of neighbors and $r$ is $130 \mathrm{~m}$. Thus, we learn that $n$ is 8.5 for the borderline density.

Figure 9 shows that our adaptive approach gives the best overall performance - except in the most dense scenarios, where the use of AODV leads to lowest delay. The use of probe packet in our adaptive scheme adds one round-trip time (RTT) to the message end-to-end delay, but the RTT is only added for nodes to which a path can be immediately established.

\subsection{San Francisco Cab Trace}

In addition to synthetically generated traces, we have used GPSbased real-life mobility traces of San Francisco taxi cabs [1]. This data set contains GPS coordinates of approximately 500 taxis collected over 30 days in the San Francisco Bay Area. For our studies, we picked a trace file from downtown San Francisco (area dimensions: $5700 \mathrm{~m}$ times $6600 \mathrm{~m}$ ) with $116 \mathrm{cabs}$, tracked over a period of 3600 seconds. One of the main reasons why we chose to use this trace was its high resolution, node positions are recorded frequently enough to provide location information for the used radio models.

Figure 10 shows the simulation results with the mobility trace recoded from a real-life sparse scenario. We can see that epidemic routing gives the best performance, in terms if delivery ratio and delay. However, the cost of using epidemic routing comes as a heavy load on node buffer resources, which can result buffer space to become a bottleneck. Our adaptive scheme shows similar performance to epidemic routing in this mobility scenario. This is due to due to relatively sparse network that leads most of the time

\footnotetext{
${ }^{2}$ The probe packet is sent using AODV and end-to-end transport.

${ }^{3}$ The averaging weight for both link lifetime and the number of neighbors is 0.02 .
}

using DTN for which epidemic routing is used. AODV was used only rarely, only for one percent of all messages and this simulation serves mostly as a confirmation that our adaptive scheme can select appropriately in sparse scenarios.

\subsection{Helsinki Synthetic Trace}

A more dense mobility trace was obtained in a synthetic fashion: the map of Helsinki city centre (area dimensions: $4500 \mathrm{~m}$ times $3400 \mathrm{~m}$ ) was used as input for the ONE simulator [6] and 116 nodes were configured to move around Helsinki city centre according to shortest path map-based movement model, i.e., the nodes were moving between selected points of interest. Node velocity was uniformly distributed between $0.7 \mathrm{~m} / \mathrm{s}$ and $1.4 \mathrm{~m} / \mathrm{s}$ (walking speed) and pause length between 0 and $120 \mathrm{~s}$. The number of nodes was 116 and the trace duration was 3600 seconds. This mobility model provides a dense network scenario for our evaluation purposes in comparison to the aforementioned San Francisco taxi scenario, which is relatively sparse.

Figure 11 shows results with the dense network. We can now observe some performance differences between pure epidemic and adaptive mechanisms. In the adaptive scheme, AODV is now used for about five percent of all messages. The favorable effect of using adaptive mechanism (and thus AODV when suitable) can be seen as lower buffer occupancy, higher delivery ratio and lower end-to-end delay. Again, the observation is that pure AODV gives the worst performance.

\subsection{Discussion}

The simulation results with random waypoint, real-life and synthetic mobility traces, seem to confirm that our algorithm works as intended. The adaptive approach gives better performance than pure AODV or pure DTN - except in the most dense scenarios, where the use of AODV leads to lowest delay. The benefit of using pure AODV instead of our adaptive approach comes from missing need to send probe packets, which always add one RTT to the endto-end delay.

The benefits of using the adaptive algorithm are not as pronounced in the real-life and synthetic trace scenarios as in the synthetic random waypoint scenario. This is because the relatively sparse traces lead the adaptive scheme to choose DTN very often and AODV, which results in resource savings is rarely chosen. However, we expect that some real-life scenarios are more clustering, which would lead to using AODV more often than in the scenarios we used in the above simulations.

\section{CONCLUSION AND NEXT STEPS}

In this paper we have shown how to adaptively choose between AODV (with TCP as transport protocol) and DTN routing (with the bundle protocol for transport). Our simulation results confirm that AODV needs a dense enough network in order to ensure low delay and high delivery ratio, where as DTN can be used to increase message delivery success in sparse scenarios.

Our simulations with accurate wireless radio models show that performance of the epidemic routing suffers from too dense networks as the nodes run out of bandwidth, due to too many message copies and wireless link layer collisions, and the result is seen as increased delays and decreased delivery ratios. Naturally, if there is enough bandwidth and buffer space this is not a problem, but the network density sets limits simultaneous transfers.

Even though epidemic routing leads to highest delivery probability in a perfect world (bandwidth and buffer space are not bottlenecks), binary spray and wait is a more reasonable choice for DTN routing protocol. Naturally, the number of message copies 


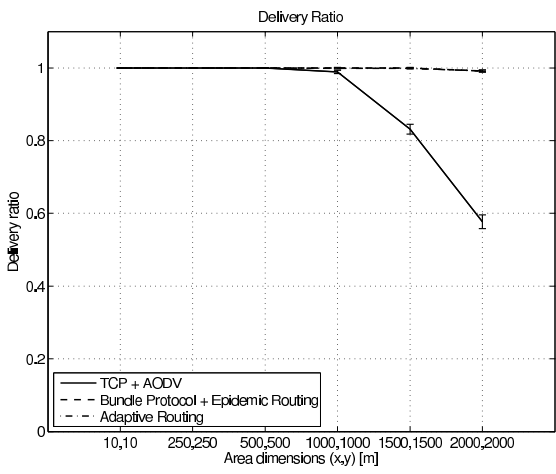

(a) Delivery ratio.

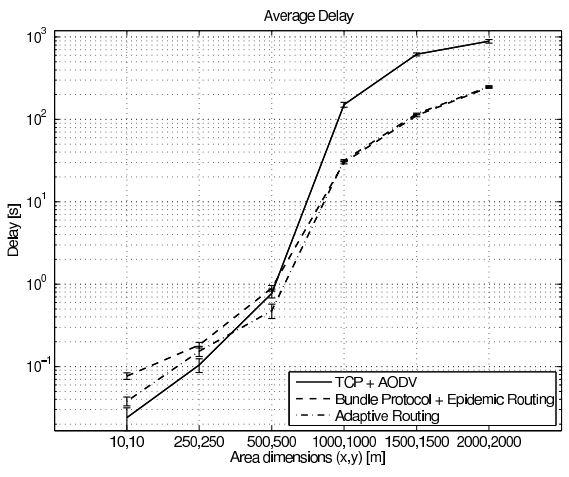

(b) Average delay.

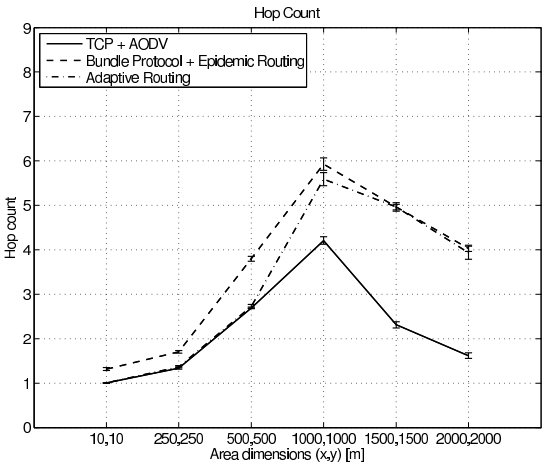

(c) Average hop count.

Figure 9: Random waypoint: 40 nodes, $10 \mathrm{kB}$ messages, fast mobility (max. $20 \mathrm{~m} / \mathrm{s}$ ), adaptive routing.

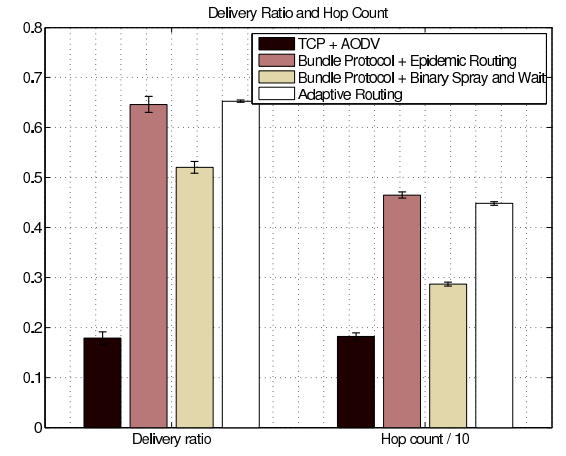

(a) Delivery ratio and hop count.

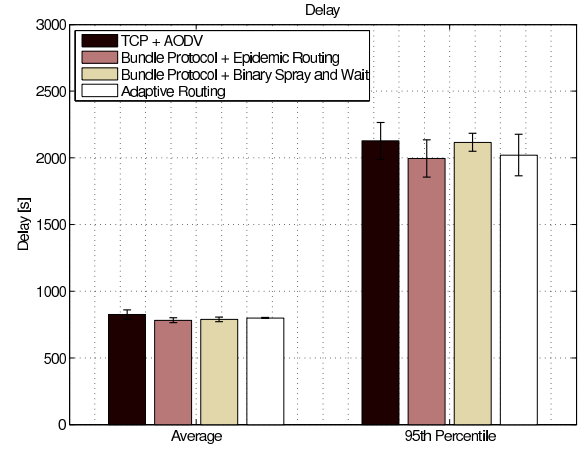

(b) Delay.

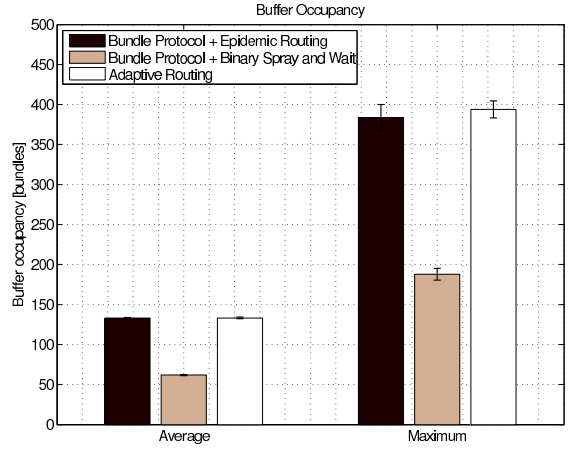

(c) Buffer occupancy.

Figure 10: Real trace, $10 \mathrm{kB}$ messages, 116 nodes.

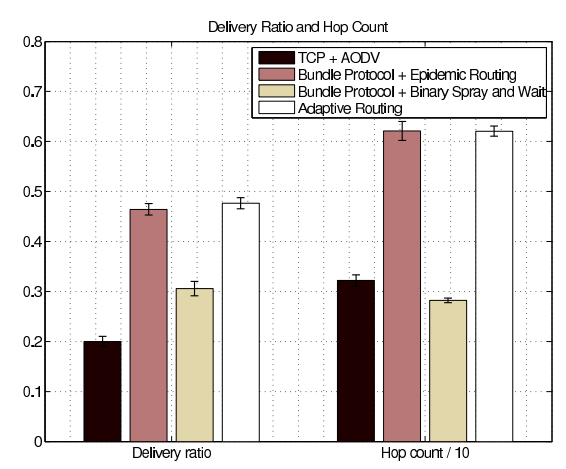

(a) Delivery ratio and hop count.

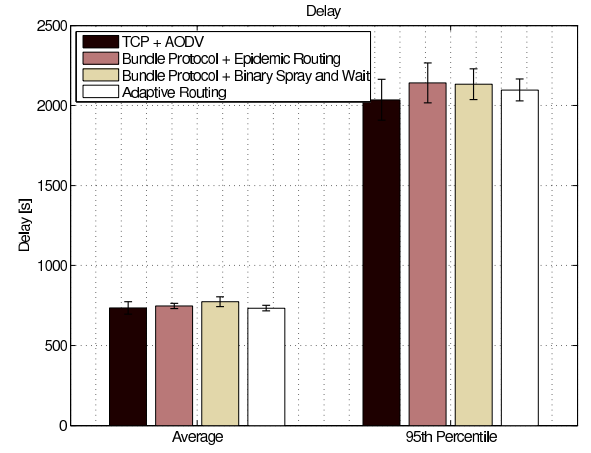

(b) Delay.

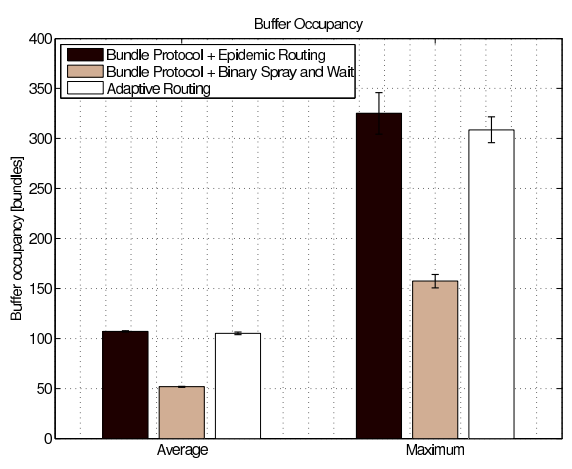

(c) Buffer occupancy.

Figure 11: Synthetic trace, $10 \mathrm{kB}$ messages, 116 nodes.

should be carefully selected. Moreover, sending fewer copies is more energy-efficient and helps to avoid wireless link layer collisions in dense scenarios.

Independent of the DTN routing protocol used, some kind of congestion control is needed. In the simplest form, this mechanism can be implemented so that in addition to the bundle identifiers the Hello message would include the bundle storage congestion level.
No bundles would be sent to neighbors with overloaded buffers. The details of this mechanism are for further study.

The selection between TCP (with MANET routing) and the bundle protocol (with DTN routing) should depend at least on node density, message size and node velocity. The benefit of using these metrics comes from the property that they can be locally measured or estimated per node, and no individual state keeping for other 
nodes needs to be done. Message size and own speed are naturally known while node density can be estimated by keeping an aggregate measure over the nodes encountered [16].

Moreover, we believe that the use of broadcast (instead of multiple unicast messages) could improve the performance of epidemic routing in a dense wireless network, where bandwidth (and not the buffer space) is the bottleneck. This is for further study.

\section{REFERENCES}

[1] Cabspotting. http: / / cabspotting.org.

[2] Delay Tolerant Networking Research Group. http: / / www. dtnrg.org.

[3] Z. Haas and T. Small. A new networking model for biological applications of ad hoc sensor networks. IEEE/ACM Transactions on Networking, 14(1):27-40, February 2006.

[4] S. Heimlicher, R. Baumann, M. May, and B. Plattner. The Transport Layer Revisited. In Proceedings of the 2nd International Conference on Communication Systems Software and Middleware, IEEE COMSWARE 2007, Bangalore, India, January 2007.

[5] S. Heimlicher, M. Karaliopoulos, H. Levy, and M. May. End-to-end vs. Hop-by-hop Transport under Intermittent Connectivity. In Proceedings of ICST Autonomics 2007, Rome, Italy, October 2007.

[6] A. Keränen, J. Ott, and T. Kärkkäinen. The ONE Simulator for DTN Protocol Evaluation. In SIMUTools '09: Proceedings of the 2nd International Conference on Simulation Tools and Techniques, New York, NY, USA, 2009. ICST.

[7] H. Ochiai, K. Shimotada, and H. Esaki. Dtipn: delay tolerant ip networking for opportunistic network applications. In MobiOpp '10: Proceedings of the Second International Workshop on Mobile Opportunistic Networking, pages 65-71, New York, NY, USA, 2010. ACM.

[8] U. of Padova. dei80211mr: a new 802.11 implementation for NS-2. http:

//www. dei. unipd.it/wdyn/?IDsezione=5090.

[9] J. Ott, D. Kutscher, and C. Dwertmann. Integrating DTN and MANET routing. In Proceedings of the 2006 ACM SIGCOMM Workshop on Challenged Networks, Pisa, Italy, September 2006.

[10] C. Perkins. Ad Hoc Networking. Addison-Wesley, 2001.

[11] C. Perkins, E. Belding-Royer, and S. Das. Ad hoc On-Demand Distance Vector (AODV) Routing. RFC Experimental 3561, Internet Engineering Task Force, July 2003.

[12] C. Perkins and E. Royer. Ad-hoc On-Demand Distance Vector Routing. In Proceedings of the 2nd IEEE Workshop on Mobile Computing Systems and Applications, pages 90-100, New Orleans, LA, USA, February 1999.

[13] M. Petrova, L. Wu, M. Wellens, and P. Mähönen. Hop of No Return: Practical Limitations of Wireless Multi-Hop Networking. In REALMAN 2005, 2005.

[14] N. Sadagopan, F. Bai, B. Krishnamachari, and A. Helmy. Paths: analysis of path duration statistics and their impact on reactive manet routing protocols. In MobiHoc '03:

Proceedings of the 4th ACM international symposium on Mobile ad hoc networking \& computing, pages 245-256, New York, NY, USA, 2003. ACM.

[15] K. Scott and S. Burleigh. Bundle Protocol Specification.
RFC Experimental 5050, Internet Engineering Task Force, November 2007.

[16] T. Spyropoulos, K. Psounis, and C. Raghavendra. Spray and wait: an efficient routing scheme for intermittently connected mobile networks. In Proceedings of the 2005 ACM SIGCOMM Workshop on Delay-Tolerant Networking, pages 252-259, Philadelphia, PA, USA, August 2005.

[17] UCB/LBNL/VINT. Network Simulator - ns (version 2). http://www.isi.edu/nsnam/ns.

[18] A. Vahdat and D. Becker. Epidemic routing for partially connected ad hoc networks. Technical report, Duke University, April 2000.

[19] B. Walker, J. Glenn, and T. Clancy. Analysis of Simple Counting Protocols for Delay-Tolerant Networks. In Proceedings of CHANTS'07, Montréal, Québec, Canada, September 2007.

[20] J. Whitbeck and V. Conan. HYMAD: Hybrid DTN-MANET Routing for Dense and Highly Dynamic Wireless Networks. In Proceedings of WoWMoM Workshop on Autonomic and Opportunistic Communications, Kos, Greece, June 2009. 